\title{
Corrigendum: Effect of Black Tea Extract and Thearubigins on Osteoporosis in Rats and Osteoclast Formation in vitro
}

\author{
Qingqing Liang ${ }^{1,2,3}$, Ming $\mathrm{Lv}^{1,2,3}$, Xiaojuan Zhang ${ }^{1,2,3}$, Jun $\mathrm{Hu}^{4}$, Ying $\mathrm{Wu}^{4}$, Yewei Huang ${ }^{1,2,4 *}$, \\ Xuanjun Wang ${ }^{1,2,4,5 *}$ and Jun Sheng ${ }^{1,2,4,5 *}$ \\ ${ }^{1}$ Key Laboratory of Pu-er Tea Science, Ministry of Education, Yunnan Agricultural University, Kunming, China, ${ }^{2}$ Tea Research \\ Center of Yunnan, Kunming, China, ${ }^{3}$ College of Food Science and Technology, Yunnan Agricultural University, Kunming, \\ China, ${ }^{4}$ College of Science, Yunnan Agricultural University, Kunming, China, ${ }^{5}$ State Key Laboratory for Conservation and \\ Utilization of Bio-Resources in Yunnan, Kunming, China
}

Keywords: osteoporosis, black tea extract, thearubigins, osteoclast, osteoclastogenesis

\section{OPEN ACCESS}

Edited and reviewed by:

Geoffrey A. Head,

Baker Heart and Diabetes

Institute, Australia

*Correspondence:

Yewei Huang

lichuangyewei100@163.com

Xuanjun Wang

wangxuanjun@gmail.com

Jun Sheng

shengj@ynau.edu.cn

Specialty section

This article was submitted to Integrative Physiology,

a section of the journal

Frontiers in Physiology

Received: 10 January 2020

Accepted: 07 February 2020

Published: 25 February 2020

Citation:

Liang Q, Lv M, Zhang X, Hu J, Wu Y,

Huang $Y$, Wang $X$ and Sheng J (2020)

Corrigendum: Effect of Black Tea

Extract and Thearubigins on

Osteoporosis in Rats and Osteoclast

Formation in vitro.

Front. Physiol. 11:136

doi: 10.3389/fphys.2020.00136

\section{A Corrigendum on}

Effect of Black Tea Extract and Thearubigins on Osteoporosis in Rats and Osteoclast Formation in vitro

by Liang, Q., Lv, M., Zhang, X., Hu, J., Wu, Y., Huang, Y., et al. (2018). Front. Physiol. 9:1225. doi: $10.3389 /$ fphys.2018.01225

In the original article, there was a mistake in Figure 2 as published. Panels 2 and 3 (cortical bone tissue stained with H\&E for Model and XLGB group, respectively) of Figure 2E in this paper are the same images as panels 2 and 3 of Figure 4D in Wang et al. (2018). Based on the 3R (Reduction, Replacement, and Refinement) principle of experimental animals, the authors simultaneously and systematically evaluated the pharmacological effects of Dendrobium officinale Orchid extract, black tea extract, and thearubigins in preventing osteoporosis using the same batch of ovariectomized (OVX) female rats as animal model of postmenopausal osteoporosis in the animal experiment study. They then collected the data and published two articles mentioned and this is how this error was introduced. The corrected Figure 2 appears below.

The authors apologize for this error and state that this does not change the scientific conclusions of the article in any way. The original article has been updated.

\section{REFERENCES}

\footnotetext{
Wang Q, Zi C-T, Wang J, Wang Y-N, Huang Y-W, Fu X-Q, et al. (2018). Dendrobium officinale orchid extract prevents ovariectomy-induced osteoporosis in vivo and inhibits RANKL-induced osteoclast differentiation in vitro. Front. Pharmacol. 8:966. doi: 10.3389/fphar.2017.00966
}

Copyright (c) 2020 Liang, Lv, Zhang, Hu, Wu, Huang, Wang and Sheng. This is an open-access article distributed under the terms of the Creative Commons Attribution License (CC BY). The use, distribution or reproduction in other forums is permitted, provided the original author(s) and the copyright owner(s) are credited and that the original publication in this journal is cited, in accordance with accepted academic practice. No use, distribution or reproduction is permitted which does not comply with these terms. 


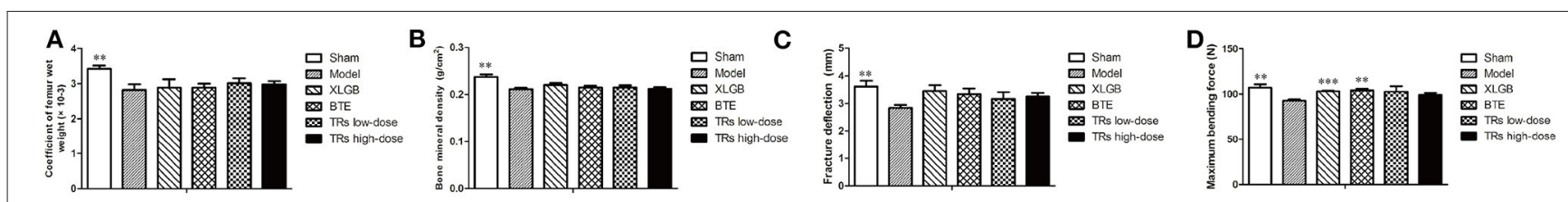

E
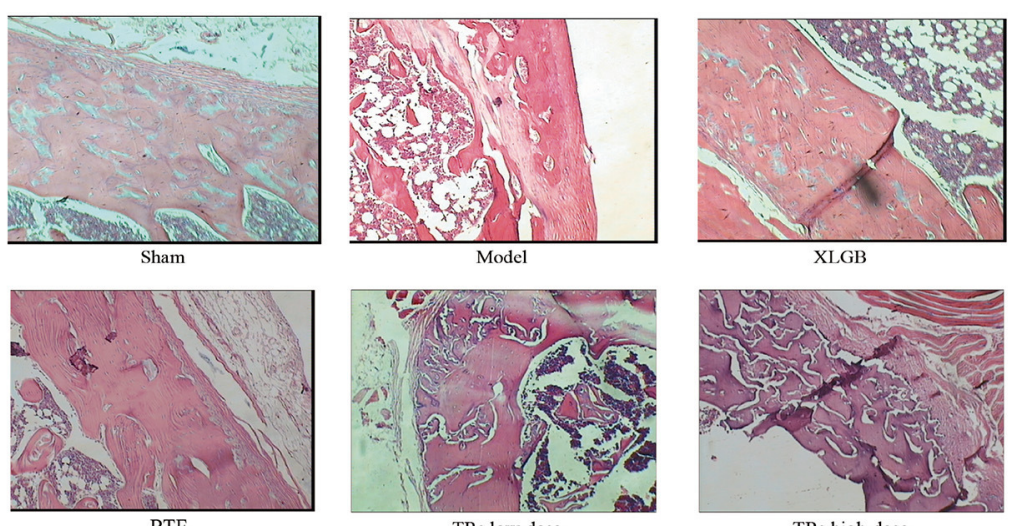

$\mathbf{F}$

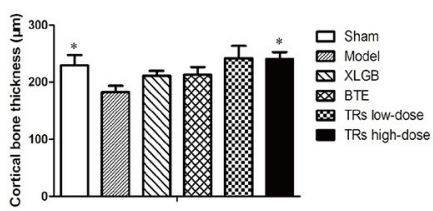

FIGURE 2 | The effect of black tea extract (BTE) and thearubigins (TRs) on bone quality in ovariectomized (OVX) rats. (A) Femur wet weight coefficient, (B) bone mineral density, (C) femoral fracture deflection, (D) maximum bending force for each treatment group. (E) The cortical bone tissue was examined by hematoxylin and eosin (H\&E) staining (magnification $\times 400$ ) and (F) cortical bone thickness counts, statistical analysis. All data are presented as mean \pm SEM ( $n=10)$. "Independent Samples T-Test" and "One way ANOVA" were used for the comparison of sham vs model group and model vs treatment groups using pooled variance, respectively. ${ }^{*} p<0.05,{ }^{\star *} p<0.01$, and ${ }^{* \star *} p<0.001$ vs model group. 